\title{
Estudo de Validade de uma Escala de Desempenho em Tecnologias para Estudantes
}

\author{
Validade da Escala de Desempenho em Tecnologias
}

\author{
Maria Cristina Rodrigues Azevedo Joly \\ Ronei Ximenes Martins
}

\section{Resumo}

O presente estudo buscou evidências de validade para a Escala de Desempenho em Tecnologias (EDETEC) para estudantes. Participaram 463 alunos com idade variando entre 15 e 60 anos $(M=22 ; D P=6,9)$, sendo $53,1 \%$ do sexo feminino. A maioria freqüentava escolas públicas (84,9\%), sendo $47,1 \%$ matriculados no ensino médio e $52.9 \%$ universitários ingressantes. A EDETEC com 95 itens foi respondida individualmente em aplicação coletiva. A análise das características psicométricas revelou boa consistência interna $( \pm=0,96)$ e a precisão interna, verificada pelo método das metades, foi de $0,87 \mathrm{com}$ homogeneidade dos itens. A análise fatorial indicou a presença de 3 fatores que explicam $47,25 \%$ da variância. Foram eliminados 39 itens, com comunalidade menor que 0,40 , para a constituição da primeira versão.

Palavras-chave: Construção de testes; Psicometria; Desempenho teste.

\section{Validity study of technology performance scale for students}

\begin{abstract}
This study aims to evaluate the psychometric characteristics of the Escala de Desempenho em Tecnologias (EDETEC). 463 students with age between 15 and 60 years $(M=22 ; D P=6,9)$ have participated, $53,1 \%$ female, $84.9 \%$ from public schools. EDETEC, which has 95 Likert items with a fouranswer choice each, was answered individually. The results showed a good internal consistency $( \pm=0,97)$. The internal reliability, verified by the splithalf method, was 0,87 and it showed that the items were homogenous. The factor analysis found three factors that explained $47,25 \%$ of the variations. So 39 items (with communalities $<0,40$ ) were eliminated to the first version.
\end{abstract}

Keywords: Test construction; Psychometrics; Performance test

\section{Estudio de validez de una escala de desempeño en tecnología para estudiantes}

\section{Resumen}

Este estudio ha buscado evidencias de validez para la Escala de Desempenho em Tecnologías (EDETEC) para estudiantes. Han participado 463 alumnos con edades variando de entre 15 y 60 años $(m=22, d p=6,9)$, siendo $53,1 \%$ del sexo femenino. La mayor parte frecuentaba escuelas públicas (84,9\%) siendo $47,1 \%$ matriculados en la enseñanza secundaria y $52,9 \%$ universitarios iniciantes. La EDETEC, con 95 ítems, ha sido respondida de forma individual y en aplicación colectiva. El análisis de las características psicométricas ha mostrado una buena consistencia interna $( \pm=0,96)$ y la precisión interna, verificada por el método de las mitades ha sido de 0,87 con homogeneidad de los ítems. El análisis factorial ha indicado la presencia de 3 factores que explican $47,25 \%$ de la variancia. Para la construcción de la primera versión han sido eliminados 39 ítems con comunalidad menor a 0,40.

Palabras clave: Construcción de tests, Psicometría; Desempeño test. 


\section{Introdução}

As constantes mudanças nos tipos e mídias que produzem e distribuem a informação para a sociedade definem o uso de habilidades específicas para acessar, selecionar e avaliar a informação em multimídia (visual, eletrônica e digital, principalmente) bem como sua utilização (Hobbs, 2006). A inserção de tais mídias, tanto como estratégia de ensino quanto como recurso de acesso à informação, determina a necessidade de que os estudantes apresentem habilidades para se apropriarem das Tecnologias da Informação e Comunicação (TIC), especialmente no ambiente escolar (Howard, 2002; Moran, Masetto \& Behrens, 2003; Joly \& Silveira, 2003; Meade \& Dugger, 2004; Leu, Mallette, Karchmer, \& KaraSoteriou, 2005; entre outros).

Destacam-se também a adaptabilidade frente às inovações tecnológicas, criatividade para desenvolver soluções e produtos novos, flexibilidade para atuar em equipe, solucionar problemas e respeitar as diferenças no relacionamento interpessoal. Ao lado disso, planejar ações de modo estratégico, lógico, organizado e racional sendo capaz de comunicar-se por meio da linguagem oral, escrita, plástica e multimídia com criticidade e ética são alguns outros comportamentos básicos para que haja a adaptação às novas tecnologias (Tyler-Wood, Cerego \& Holcomb, 200I).

Nesse sentido, por um lado, questões de pesquisa referentes a aspectos psicológicos ligados à percepção, avaliação e atitudes frente às novas TIC têm sido investigadas (Duvel \& Pate, 2003). Escalas que buscam mensurar variáveis psicológicas tais como ansiedade (e.g., Computer Anxiety Scale, Igbaria \& Chakrabarti 1990), atitude (e.g., Computer Attitude Scales, Nickell \& Pinto, 1986) ou auto-eficácia (e.g. Computer Self-efficacy (CUSE) Scale, Cassidy \& Eachus, 2002) foram utilizadas para tal fim. A análise crítica de como os recursos tecnológicos são incorporados à prática pedagógica da escola e à pesquisa de variáveis psicológicas relacionadas ao uso das tecnologias têm estimulado psicólogos educacionais e professores a realizarem estudos nessa área (Dillon \& Gabbart, 1998; Waxman, Lin, \& Michko, 2003; Bebell, O’Dwyer, Russell, \& Seeley, 2004; Vavasseur \& MacGregor, 2006 ).
Por outro lado, um dos focos de pesquisa atualmente explorados no âmbito internacional é o desempenho de estudantes em TIC. Existem vertentes diferenciadas, centrando-se em aspectos relacionados a atitudes, ansiedade e aversão (Burkett, Compton \& Burkett, 200I) ou em auto-eficácia (Cassidy \& Eachus, 2002), cuja abordagem é bastante adotada no que se refere à mensuração do desempenho pela auto-avaliação.

Penuel, Korbak e Cole (2002) consideram que o uso educacional da TIC requer habilidades cognitivas de alto nível para, por exemplo, o desenvolvimento de projetos multimídia, simulações e comunicação por diferentes mídias. Os estudiosos denominam technology literacy ou alfabetização tecnológica a competência técnica e funcional para desenvolver e aplicar recursos tecnológicos de informação e comunicação (Joly, 2004; Leu, Kinzer, Cairo \& Cammack, 2004).

Sob o aspecto técnico, Jones (1995) definiu o desempenho em tecnologia baseando-se no tipo de uso do computador - instrução baseada no computador, simulação ou pesquisa e solução de problemas. Atualmente esse desempenho está relacionado ao envolvimento do estudante e sua própria competência em usar recursos tecnológicos para aprender, buscar informação e se comunicar, adaptando-se sempre às inovações presentes na TIC ( Leu, Kinzer, Coiro \& Cammack, 2004).

No tocante à funcionalidade, as habilidades básicas de leitura, de escrita e lógica matemática devem ser adaptadas às mídias digitais (Leu \& cols., 2005). Hobbs (2006) focaliza como habilidades requisitadas para essa adaptação as relacionadas à análise, compreensão e utilização crítica de imagens (visual literacy), de informação apropriada à necessidade ( information literacy) e de multimídias (medias literacy).

No Brasil, os estudos relacionados à mensuração do desempenho em TIC são bastante restritos (Joly \& Silveira, 2003; Joly, 2004; Joly \& Martins, 2005a). Existe escassez de dados quantitativos e resultados relacionados à alfabetização tecnológica no sistema educacional brasileiro. De acordo com o Instituto de Estudos e Pesquisas Educacionais Anísio Teixeira INEP (2006), no censo escolar de 2005, 91\% das 
escolas de ensino médio brasileiras contavam com computadores, sendo que $58 \%$ delas possuíam laboratórios de informática. Se considerada a dependência administrativa, $77 \%$ da rede particular oferecia laboratórios de informática contra $51 \%$ da rede pública. Pelo levantamento, $49 \%$ dos matriculados no ensino médio público em 2005 tiveram acesso a recursos de TIC. Nas instituições particulares este número eleva-se para $78 \%$. Entretanto, os dados não explicitam o número de alunos que efetivamente receberam qualificação no uso de tecnologias em suas atividades na escola e qual seu nível de habilidade nas ferramentas básicas. Também não há relato de utilização de instrumento para mensurar o desempenho.

Segundo busca concentrada em publicações entre 2000 e 2005, nas bases de dados Biblioteca Virtual de Saúde - BVS, Base de dados de artigos de periódicos nacionais em Educação da Faculdade de Educação da Universidade Estadual de Campinas - EDUBASE, Educational Resources Information Center - ERIC, PsycINFO e Scientific Electronic Library Online - SCIELO, não estão disponíveis, no Brasil, estudos específicos quanto ao desempenho de estudantes em TIC. Também não existem trabalhos publicados sobre o desenvolvimento e/ou evidencias de validade de instrumentos que visem mensurar o desempenho de alunos frente ao uso de tecnologias em situações de ensino-aprendizagem.

Considerando as perspectivas de Bandura (1989), Eastin e LaRose (2000) e de Duvel e Pate (2004) uma sondagem sobre do desempenho de alunos em TIC não necessita incorporar testes de habilidade técnica em utilizar este e aquele dispositivo ou software. Essa pode buscar padrões relacionados ao domínio de tais recursos por meio de escalas auto-avaliação. Para construir escalas que obtenham desempenho em TIC é necessário definir que habilidades são esperadas em determinado domínio. Sem um padrão de ações é difícil estabelecer parâmetros quanto ao que avaliar.

Considerando esta necessidade, alguns organismos internacionais desenvolveram padrões quanto às habilidades em tecnologia esperadas das pessoas para cada etapa de formação na escola.
Um dos padrões, utilizado pela United Nation Educational, Scientific and Cultural Organization UNESCO (2004), criado pela International Society for Technology in Education - ISTE (2000), vem sendo adotado nos EUA e em outros paises. A Europa também possui recomendações oficiais quanto às habilidades em TIC. A Rede de Informação sobre Educação da Europa EURYDICE, publica anualmente o Basic Indicators on the Incorporation of ICT into European Education Systems (UNESCO, 2004). O padrão ISTE, assim como outros, pode ser tomado como base para obtenção de dados quanto ao desempenho do professor e do aluno em tecnologias de informação e comunicação.

Ao determinar os padrões para estudantes, o ISTE definiu seis categorias, criando um modelo que conecta indicadores de desempenho com perfis desejados de alfabetização tecnológica. Os indicadores estão organizados de acordo com o nível de escolaridade dos alunos da educação básica. $O$ quadro I apresenta o desempenho esperado para um aluno que concluiu a educação básica.

Verificando a necessidade de instrumentos específicos que permitissem obtenção do desempenho de estudantes em tecnologias de informação e comunicação, na perspectiva da autoavaliação, o presente estudo objetivou buscar evidências de validade de construto para a Escala de Desempenho em Tecnologia (EDETEC). Analisou relações entre o desempenho dos participantes e a idade e o gênero e o tipo de escola que freqüentavam.

\section{Método}

\section{Participantes}

Participaram da presente pesquisa 463 alunos, $74,7 \%$ de Minas Gerais e 25,3\% de São Paulo. Dentre eles, $53,1 \%$ eram do sexo feminino e $46,9 \%$ do sexo masculino, com idade variando entre 15 e 60 anos $(M=22 ; D P=6,9)$. A maioria $(84,9 \%)$ estudava em escolas particulares, $47,1 \%$ no ensino médio e $52,9 \%$ no primeiro ano do ensino superior. 
Quadro I - Categorias e indicadores de desempenho em tecnologias de comunicação e informação para alunos concluintes do ensino médio.

\begin{tabular}{|c|c|c|}
\hline Categoria & Habilidades & Indicadores de desempenho \\
\hline $\begin{array}{l}\text { Operações } \\
\text { básicas e } \\
\text { conceitos. }\end{array}$ & $\begin{array}{l}\text { - } \text { demonstrar entendimento da } \\
\text { natureza e operação de sistemas } \\
\text { baseados em tecnologia } \\
\text { - } \quad \text { ser proficiente no uso das tecnologias } \\
\text { mais acessíveis. }\end{array}$ & $\begin{array}{l}\text { saber escolher dentre os sistemas, recursos } \\
\text { e serviços disponíveis para uso. } \\
\text { usar dos dispositivos e ferramentas } \\
\text { tecnológicas comuns. } \\
\text { solucionar problemas rotineiros de } \\
\text { hardware e software. }\end{array}$ \\
\hline $\begin{array}{l}\text { Questōes } \\
\text { sociais, éticas e } \\
\text { humanas } \\
\text { relacionadas à } \\
\text { tecnologia. }\end{array}$ & $\begin{array}{l}\text { entender os problemas éticos, } \\
\text { culturais e sociais relacionados ao uso } \\
\text { (ou não) da tecnologia. } \\
\text { revelar uso responsável e desenvolver } \\
\text { atitudes positivas frente às TIC. }\end{array}$ & $\begin{array}{l}\text { - identificar capacidade, limitações e potencial } \\
\text { dos recursos tecnológicos emergentes. } \\
\text { - } \text { analisar vantagens e d esvantagens do uso de } \\
\text { TIC. } \\
\text { - demonstrar comportamentos éticos e legais } \\
\text { quanto ao uso de TIC. }\end{array}$ \\
\hline $\begin{array}{l}\text { Ferramentas de } \\
\text { produtividade. }\end{array}$ & $\begin{array}{l}\text { usar ferramentas para aprimorar } \\
\text { aprendizagem e ampliar } \\
\text { produtividade. } \\
\text { usar ferramentas para colaborar na } \\
\text { construçáo de aprimoramentos } \\
\text { tecnológicos e realizar tarefas com } \\
\text { criatividade. }\end{array}$ & $\begin{array}{l}\text { - usar tecnologias na gestão pessoal e } \\
\text { - } \quad \text { revissional de informaçãa. } \\
\text { informação online para produtividade. }\end{array}$ \\
\hline $\begin{array}{l}\text { Ferramentas de } \\
\text { comunicação. }\end{array}$ & $\begin{array}{l}\text { - usar TIC para colaborar, publicar e } \\
\text { interagir. } \\
\text { - utilizar diferentes mídias para } \\
\text { comunicação eficiente. }\end{array}$ & $\begin{array}{l}\text { - usar tecnologias na comunicação pessoal e } \\
\text { - } \quad \text { revissional. } \\
\text { informaçãa online para colaboração e } \\
\text { comunicação. }\end{array}$ \\
\hline $\begin{array}{l}\text { Ferramentas de } \\
\text { pesquisa. }\end{array}$ & $\begin{array}{l}\text { usar tecnologia para localizar avaliar e } \\
\text { coletar informações de fontes } \\
\text { variadas. } \\
\text { usar TIC para processar dados } \\
\text { coletados e comunicar resultados. } \\
\text { avaliar e selecionar novos recursos e } \\
\text { inovaçōes tecnológicas apropriadas } \\
\text { para tarefas específicas. }\end{array}$ & $\begin{array}{l}\text { avaliar opções para aprendizagem } \\
\text { continuada baseada em tecnologia, } \\
\text { incluindo educação a distância. } \\
\text { revelar uso rotineiro e eficaz de } \\
\text { recursos de informação online na } \\
\text { pesquisa e para publicação. } \\
\text { investigar e aplicar sistemas } \\
\text { especialistas, agentes inteligentes e } \\
\text { simuladores em situações de pesquisa. }\end{array}$ \\
\hline $\begin{array}{l}\text { Ferramentas de } \\
\text { resolução de } \\
\text { problemas }\end{array}$ & $\begin{array}{l}\text { usar recursos tecnológicos para } \\
\text { solucionar problemas e para } \\
\text { tomada de decisóes. }\end{array}$ & 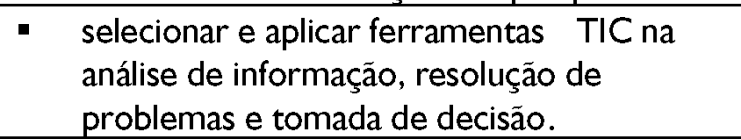 \\
\hline
\end{tabular}

Adaptado do ISTE (2000).

\section{Instrumento}

\section{Escala de Desempenho em Tecnologia -} EDETEC -Anexo I (Joly \& Martins, 2005).

Visa identificar características de desempenho, relacionadas ao domínio técnico e, também, ao uso eficaz e ético dos recursos tecnológicos como ferramentas, no que se refere à adequação de sua aplicação no cotidiano e como item de aprendizagem, na perspectiva da alfabetização tecnológica. É composta por 95 itens do tipo Likert com quatro pontos $(0=$ nunca, $I=$ algumas vezes, $2=$ muitas vezes, $3=$ sempre), podendo ser aplicada em formato impresso ou eletrônico A pontuação máxima é de 285 pontos. Trata-se de instrumento de aplicação individual ou coletiva com tempo médio de 20 minutos.

Os itens da EDETEC foram elaborados considerando-se as categorias e indicadores de desempenho em tecnologias de comunicação e 
informação para alunos concluintes do ensino básico organizada pelo ISTE (2000). Todas as categorias foram contempladas com I5 itens em média. Cada item expressa um único comportamento característico e com afirmativas positivas. A linguagem e o conteúdo dos itens foram analisados por cinco especialistas em psicometria, sendo quatro psicólogos e um profissional que atua com sistemas de informação, e 10 estudantes ( cinco concluintes do ensino médio e cinco universitários ingressantes). Houve concordância de $95 \%$ entre o grupo de especialistas e $97 \%$ no de estudantes. As modificações sugeridas foram acrescentar uma explicação como aposto ( e.g. itens $5,6,17$ ) e usar termos comuns e próprios a TIC como e- mail, download, acessar, palm, online, deletar e MSN, as quais foram acatadas.

\section{Resultados e Discussão}

Verificada a fidedignidade por meio do teste Alfa de Cronbach, constatou-se que a escala apresentou ótima consistência interna $( \pm=0,96)$. A precisão interna foi verificada pelo método das metades, e revelou homogeneidade dos itens $(0,87)$. $O$ teste de esfericidade de Bartlett indicou que a matriz de correlação não é uma matriz identidade, portanto existe correlação entre os itens avaliados ( $x 2$ [4465, $\mathrm{N}=643]=25656,292 ; p>0,000)$. A medida de adequação da amostra de Kaiser-Meyer-Olkin (KMO $=0,96$ ) indicou que é muito adequada, pelo grau de variância apresentado, para avaliar o desempenho do estudante na utilização de recursos tecnológicos na educação, conferindo validade aos resultados.

Tabela I - Distribuição dos autovalores e variação explicada por fator extraído para primeira análise fatorial dos resultados

\begin{tabular}{cccc}
\hline & & \multicolumn{2}{c}{$\%$ da variância explicada } \\
\cline { 3 - 4 } Fatores & Autovalores iniciais & Simples & Acumulada \\
1 & 27,77 & 29,24 & 29,24 \\
2 & 4,00 & 4,21 & 33,45 \\
3 & 3,16 & 3,33 & 36,78 \\
4 & 2,40 & 2,52 & 39,30 \\
5 & 2,16 & 2,27 & 41.57 \\
6 & 2,11 & 2,22 & 43,80 \\
\hline
\end{tabular}

\section{Procedimento}

O estudo foi executado com autorização dos respectivos responsáveis pelas instituições educacionais nas quais os alunos estavam matriculados. Realizou-se reunião prévia com os aplicadores, que foram orientados quanto aos procedimentos de aplicação. No momento da aplicação os alunos foram esclarecidos quanto ao objetivo da pesquisa, destacando-se que suas identidades seriam mantidas em sigilo. Em seguida, foi pedido que eles lessem o Termo de Consentimento Livre e Esclarecido. Os que consentiram receberam a EDETEC no formato impresso e responderam individualmente, sem o auxilio do aplicador, por 20 minutos em média.
A análise fatorial dos resultados obtidos foi executada utilizando-se o método Varimax de extração por fatores, considerando o critério da raiz latente para a constituição de fatores com os autovalores maiores que um. A primeira análise indicou a presença de seis fatores (Tabela I) explicando $43,8 \%$ da variância. O fator I era o que tinha maior índice de variância $(29,24 \%)$, ou seja, era o que melhor explicava a relação entre os itens da escala. A distribuição dos itens se deu de maneira que 80 representavam o Fator I, com carga fatorial variando de 0,98 a 0,23 . $O$ fator 2 recebeu 7 itens de carga fatorial entre 0,5 I e 0,32. Os fatores 3 a 6 agruparam 2 itens cada, com cargas entre 0,80 e 0,27. 
Foram então eliminados 21 itens com cargas inferiores a $0,40(2,3,5,10,13,14,20,22,24,25$, $28,32,42,46,63,76,79,81,82,91,94)$ e executada nova análise para a escala com 74 itens. O resultado apresentou distribuição em 3 fatores explicando $39,48 \%$ da variância, sendo $16,52 \%$ no fator 1 , $15,00 \%$ no fator 2 e $7,96 \%$ no fator 3 .

Avaliadas as cargas fatoriais recalculadas, observouse nova incidência de valores inferiores a 0,40 , o que permitiu a exclusão de mais $I 5$ itens $(I, 4,27,3 I, 36$, $40,43,47,51,54,64,65,83,88,95)$. Observou-se, também, que os itens 39,59 e 78 , pertencentes ao fator 2, apresentavam carga inferior a 0,45 e destoavam em termos de conteúdo dos demais. Optou-se, então, por eliminar também estes e executar a terceira análise fatorial, com os mesmos parâmetros da anterior, agora com 56 itens. A distribuição em 3 fatores manteve-se, como se observa pela análise do gráfico de Scree Plot (Figura I), porém com maior homogeneidade da variância entre eles.
A medida de adequação da amostra de KaiserMeyer-Olkin foi mantida em 0,96 e o resultado do teste Bartlett para esta análise manteve a indicação que a matriz de correlação não é uma matriz identidade ( $x 2$ [I540, $N=643]=|5| 45,536 ; p>0,000)$.

Após a exclusão dos 39 itens, houve aumento de todos os indicadores de variância da escala e a distribuição em três fatores que passaram a explicar $47,25 \%$ da variância. Esse índice é melhor que o observado com a presença de seis fatores, indicando que a retirada de itens implicou em uma melhor adequação da dimensionalidade da escala (Tabela 2).

Os itens distribuídos nos três fatores apresentaram carga significativa $(0,44$ a 0,78$)$. O Fator I concentrou os 17 itens relativos às ferramentas básicas e de comunicação, relacionadas às habilidades no uso de tecnologias para tarefas elementares de obtenção de informação, interação em rede e aprendizagem cooperativa e foi denominado ferramentas básicas e de comunicação. No que se refere ao fator 2 , denominado

Tabela 2 - Distribuição dos autovalores e variação explicada por fator extraído para terceira análise fatorial dos resultados com exclusão de 39 itens

\begin{tabular}{cccc}
\hline & & \multicolumn{2}{c}{$\%$ da variância explicada } \\
\cline { 3 - 4 } Fatores & Autovalores iniciais & Simples & Acumulada \\
1 & 9,36 & 16,72 & 16,72 \\
2 & 8,92 & 15,94 & 32,66 \\
3 & 8,17 & 14,59 & 47,25 \\
\hline
\end{tabular}

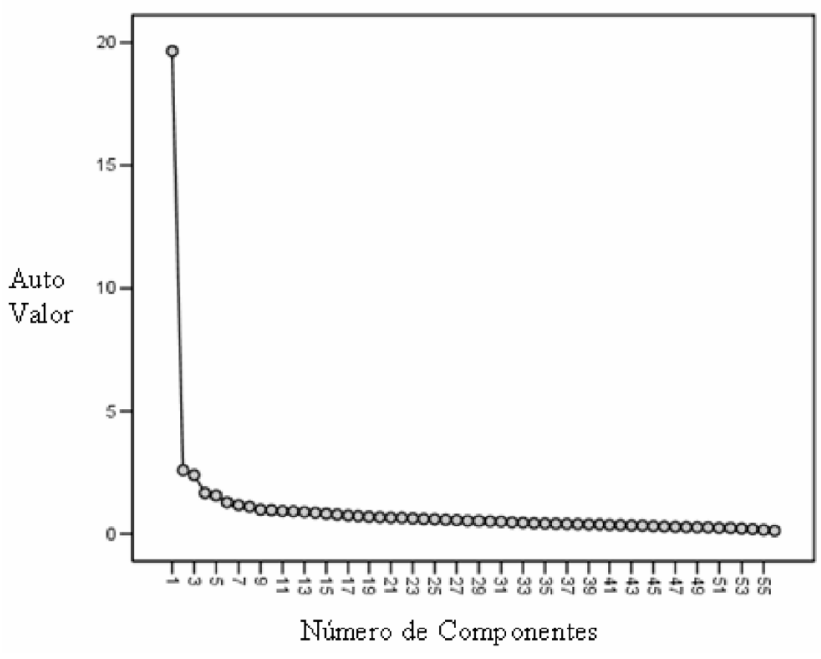

Figura I - Resultado da análise de itens pelo método Scree Plot conceitos e ferramentas de produtividade, este agrupou 20 itens que descrevem atitudes de uso produtivo do instrumental visando aprimoramento da aprendizagem de conteúdos, desenvolvimento criativo de tarefas e exposição de idéias. No fator 3 concentraram-se os 19 itens que descrevem atitudes relacionadas com a utilização avançada de recursos tecnológicos para solucionar problemas do dia-a-dia e para tomada de decisões, nomeado como ferramentas de solução de problemas. A Tabela 3 apresenta o agrupamento dos itens por fatores e suas cargas. A concentração dos itens nos três fatores corresponde ao critério estabelecido pela ISTE (2000). 
Tabela 3 - Questões ordenadas por cargas fatoriais e distribuídas em três fatores depois de retirada de 39 itens.

\begin{tabular}{crcrcr}
\hline Questões & Fator 1 & Questões & Fator 2 & Questões & Fator 3 \\
\hline 69 & 0,78 & 56 & 0,67 & 11 & 0,64 \\
38 & 0,76 & 55 & 0,62 & 48 & 0,62 \\
21 & 0,73 & 15 & 0,61 & 52 & 0,62 \\
90 & 0,68 & 93 & 0,59 & 29 & 0,60 \\
86 & 0,66 & 67 & 0,58 & 08 & 0,59 \\
71 & 0,65 & 33 & 0,55 & 30 & 0,59 \\
62 & 0,63 & 44 & 0,55 & 17 & 0,57 \\
35 & 0,62 & 16 & 0,53 & 23 & 0,57 \\
58 & 0,60 & 72 & 0,52 & 34 & 0,57 \\
70 & 0,60 & 53 & 0,51 & 68 & 0,56 \\
77 & 0,60 & 74 & 0,51 & 12 & 0,55 \\
87 & 0,55 & 57 & 0,50 & 45 & 0,55 \\
66 & 0,54 & 92 & 0,50 & 07 & 0,52 \\
73 & 0,52 & 19 & 0,49 & 85 & 0,48 \\
61 & 0,51 & 75 & 0,49 & 06 & 0,47 \\
80 & 0,45 & 50 & 0,48 & 26 & 0,46 \\
60 & 0,41 & 41 & 0,47 & 84 & 0,46 \\
& & 49 & 0,45 & 18 & 0,44 \\
& & 09 & 0,44 & 89 & 0,44 \\
& & 37 & 0,44 & & \\
\hline
\end{tabular}

Verificou-se que os três fatores se correlacionaram positiva e significativamente. Os índices obtidos para as correlações foram, para fatores I com 2, $r=0,76$ $(p>0,000)$ e para I com 3 e 2 com $3, r=0,75$ $(p>0,000)$. A correlação dos fatores com o escore total apresentou índices superiores a 0,90 ( $p>0,000)$. Também a consistência interna para cada fator apresentou índices $\pm=0,90$ (fator I) e $\pm=0,9 \mathrm{I}$ (fatores 2 e 3), confirmando os bons indicadores de precisão após a exclusão dos itens. A EDETEC ficou com 56 itens os quais foram seqüenciados na escala por sorteio aleatório. A pontuação máxima passou a ser de 168 pontos, sendo 5I, 60 e 57, respectivamente para os fatores I, 2 e 3.

Quanto à relação entre gênero dos participantes e dependência administrativa de matrícula do aluno no que se refere ao desempenho em TIC, a análise inferencial por meio do teste $t$ Sudent revelou que existe diferença em relação ao escore total, considerado o tipo de escola $(t[46 I]=2,57 ; p<0,01)$. Os alunos de escolas particulares $(M=87,38 ; \quad D P=35,72)$ apresentaram desempenho superior aos de escolas públicas $(M=75,74 ; D P=34,47)$. Também revelou diferença quando considerado o gênero $(t[46 I]=-4,44$; $p=0,00)$. Os participantes do sexo masculino apresentaram desempenho superior $(M=93,49$; $D P=35,34)$ ao do feminino $(M=78,67 ; D P=36,34)$. As diferenças sugerem que os alunos do sexo masculino que estudam na rede particular demonstram estarem mais preparados para o uso de TIC.

Tal observação foi corroborada isolando-se um subgrupo $(\mathrm{N}=118)$ com escore total pertencente ao quartil 75 (valores entre 113 e 164 pontos no escore total). Destes, $94 \%$ são de escolas particulares e $60 \%$ são do sexo masculino. A diferença quanto à dependência administrativa pode ter relação com fatos descritos nos indicadores do INEP (2006) visto que dos matriculados no ensino médio em 2005, 78\% dos alunos das instituições particulares tiveram acesso a recursos de TIC enquanto o índice foi de $49 \%$ nas escolas públicas. Especificamente sobre a diferença em relação ao sexo, o resultado está de acordo com o que foi obtido por Cassidy e Eachus (2002) que verificaram melhor desempenho dos participantes do sexo masculino em uma escala de auto-eficácia para usuários da Internet. 


\section{Considerações Finais}

A avaliação das qualidades psicométricas da Escala de Desempenho em Tecnologias indicou que essa apresenta evidências de validade de construto e bom indicador de precisão. Isso a coloca como instrumento a ser utilizado para avaliar os estudantes que tanto utilizam tecnologias de informação e comunicação em suas estratégias educacionais para o aprendizado, quanto ao desempenho daqueles que realizam cursos de capacitação na área.

Cabe destacar a importância da realização de outros estudos que possam ampliar as evidências de validade do instrumento. Para tal, sugere-se que seja analisado o desempenho de estudantes que usam regularmente as tecnologias como estratégia educacional comparado ao dos que usam pouco frequentemente. Verificar, também, a opinião de psicólogos escolares, coordenadores ou tutores que orientam atividades que utilizam as TIC em atividades educacionais sobre o desempenho dos alunos, comparada aos resultados obtidos por meio da aplicação da EDETEC.

É relevante, ainda, investigar as possíveis relações entre o desempenho com outras variáveis como personalidade, inteligência, criatividade, por exemplo. Além disso, estudos devem ser realizados com maior homogeneidade de participantes quanto à idade para se verificar o efeito deste aspecto em relação ao desempenho no uso de tecnologias em atividades de aprendizagem.

\section{Referências}

Bandura, A. (1989). Human agency in social cognitive theory. American Psychologist, 44, II 75-II 85.

Bebell, D., O'Dwyer, L., Russell, M., \& Seeley, K. (June, 2004). Estimating the effect of computer use at home and in school on student achievement. Paper present at National Educational Computing Conference, New Orleans, LA.

Burkett, W. H., Compton, D. M., \& Burkett, G.G., (200I). An examination of computer attitudes, anxieties, and aversions among diverse college populations: issues central to understanding information sciences in the new millennium. Informing Sciences, 4(3), 77-85.
Cassidy, S.,\& Eachus, P. (2002). Developing the computer selfefficacy scale (CUSE): investigating the relationship between computer self-efficacy, gender and experience with computers. Journal of Educational Computer Research, 26, 169-189.

Dillon, A., \& Gabbarb, R. (1998). Hypermedia as an educational technology: a review of the quantitative research literature on learner comprehension, control, and style. Review of Educational Research, 68, 322-349.

Duvel, C.,\& Pate, S. (2003). Computer knowledge: report from a student self-evaluation. Journal of Industrial Technology, 20(I), 2-16

Eastin, M.S., \& LaRose, R. (2000). Internet self-efficacy and the psychology of the digital. divide. Journal of Computer Mediated Communication, 6(I). Recuperado em 05 de jun. 2006: http://jcmc.indiana.edu/vol6/issuel/eastin.html

Eachus, P., \& Cassidy, S. (2006). Development of the Web Users Self-efficacy Scale (WUSE). Issues in Informing Science and Information Technology, 3(I). 199-209.

Hobbs, R. (2006). Multiple visions of multimedia literacy: emerging areas of synthesis. Em M. C. McKenna, L. D. Labbo, R. D. Kieffer, \& D. Reinking. International handbook of literacy and technology (vol. II, pp. 15-28). Mahwah, NJ: Lawrence Erlbaum Associates Publishers.

Howard, J. (2002). Technology-Enhanced Project-Based Learning in Teacher Education: addressing the Goals of Transfer. Journal of Technology and Teacher Education, 10, 343, 364.

Igbaria, M., \& Chakrabarti, A. (1990). Computer anxiety and attitudes towards microcomputer use. Behavior and Information Technology, 9, 229-24I.

Instituto de Estudos e Pesquisas Educacionais Anísio Teixeira (2006). Censo escolar da Educação Básica. Recuperado em 23 de maio de 2006 de http://www.inep.gov.br.

International Society for Technology in Education (2000). National Educational Technology Standards for Students. Eugene: ISTE.

Joly, M. C. R. A. (2004). Evidências de validade de uma escala de desempenho docente em informática educacional. PsicoUSF, 9, I73-180.

Joly, M.C.R.A., \& Martins, R. X. (2005a). Estudo de validade de escala de desempenho em informática educacional para professores. Avaliação Psicológica, 4, I05-II 5.

Joly, M.C.R.A., \& Martins, R. X. (2005b). Escala de desempenho em tecnologias - EDETEC. Pesquisa em desenvolvimento. Itatiba, Universidade São Francisco. 
Joly, M.C.R.A., \& Silveira, M.A. (2003). Avaliação preliminar do Questionário de Informática Educacional (QIE). Psicologia em Estudo, 8, 85-92.

Jones, S. (1995). Personal computers and gifted students. Teaching exceptional children, 27(3), 80-8I.

Leu, D. J., Kinzer, C. R., Cairo, J. L., \& Cammack, D.W. (2004). Toward a theory of new literacies emerging from the Internet and other information and communication technologies. Em R. B. Ruddell, \& N J. Unrau (Orgs), Theorical models and processes of reading (p. 1570-1613). Newark,DL:. International Reading Association.

Leu, D.J, Mallette, M.H, Karchmer, R.A., \& Kara-Soteriou, J. (2005). Innovative approaches to literacy education: using the internet to support new literacies. Newark, DL: International Reading Association.

Meade, S.D., \& Dugger, W.E. (2004). Reporting on the status of technology education in the U.S. The Technology Teacher, 64(2), 29-35.

Moran, J. M., Masetto, M. T. \& Behrens, M. A. (2003). Novas tecnologias e mediação pedagógica. ( $7^{\mathrm{a}} \mathrm{Ed}$.). Campinas,SP: Papirus.
Nickell G.S., \& Pinto, J.N. (1986). The computer attitude scale. Computers in human behavior, 2, 30I-306.

Penuel, W. K., Korbak, C., \& Cole, K. A. (2002). Designing assessments for student multimedia projects. Learning \& Leading with Technology, 29(5), 47-53.

Tyler-wood, T., Cerejo, M. V. P., \& Holcomb, T. (200I). Technology skills among gifted students. Journal of Computing in Teacher Education, 18 (2), 57-60.

UNESCO, United Nations Educational, Scientific and Cultural Organization, División de

Educación Superior (2004) Las tecnologías de la información y la comunicación en la formación docente. Guía de planificación. Montevideo:Trilce.

Vavasseur, C. B.,\& MacGregor, S. K.(July, 2006). Online Communities of Practice: Teachers and Principals Reforming Instruction. Paper present at the National Educational Computing Conference, San Diego, Ca.

Waxman, H. C., Lin, M., \& Michko, G. M. ( 2003). A metaanalysis of the effectiveness of teaching and learning with technology on student outcomes. Naperville, II: Learning Point Associates. 


\section{Anexo I -ESCALA DE DESEMPENHO EM TECNOLOGIAS- EDETEC (versão preliminar)}

\section{Maria Cristina Rodrigues Azevedo Joly Ronei Ximenes Martins}

A Escala de Desempenho em Tecnologias para Educação tem por objetivo verificar o perfil do aluno quanto ao uso de tecnologias de informação e comunicação no cotidiano e também como item de aprendizagem, na perspectiva da alfabetização tecnológica.

I. Sei consultar livrarias virtuais.

2. Faço compra de livros e outros recursos que necessito para aprender usando a Internet.

3. Eu crio novos programas de computador para resolver problemas.

4. Sei utilizar bibliotecas virtuais.

5. Procuro por vídeos ou filmes relacionados com o que estou estudando, quando não consigo compreender a matéria apenas com minhas anotações ou com os livros.

6. Sei identificar quais recursos informatizados tenho disponíveis para usar no meu dia-a-dia.

7. Tento resolver problemas de estudo usando o computador.

8. Sei escolher equipamentos que facilitem a realização de tarefas.

9. Sei usar Palm.

10. Uso telefonia via Internet.

II. Penso em soluções novas, diferentes das que estava desenvolvendo, quando uso recursos tecnológicos na resolução de problemas.

12. Procuro na Internet, sites relacionados com o que estou estudando, quando não consigo compreender a matéria.

13. Jogo pela Internet

14. Evito enviar spam.

15. Faço download de filmes e/ou musicas.

16. Sei avaliar se um site é seguro.

17. Assistir a vídeos ou filmes ligados ao assunto que estudo me faz compreender melhor.

18. Uso o computador para fazer simulações.

19. Sei detalhar a configuração dos equipamentos de informática mais comuns.

20. Penso sobre as dificuldades que as pessoas têm por não saberem usar os recursos tecnológicos.

21. Recebo e-mails.

22. Considero que os computadores influenciam a vida das pessoas.

23. Sei realizar atividades voltadas para solução de problemas usando a informática.

24. Assisto TV por assinatura.

25. Eu participo de projetos de pesquisa para desenvolver tecnologias.

26. Sei encontrar publicações científicas na Internet.

27. Evito fazer cópias, para outras pessoas, de dvds que comprei.

28. Produzo apresentações em multimídia.

29. Sei identificar o melhor recurso tecnológico de que disponho para resolver um problema.

30. Sei avaliar os recursos tecnológicos disponíveis na escola.

31. Participo de conferências com pessoas de outras escolas através de redes de telecomunicação.

32. Penso sobre as influências que as telecomunicações trazem para as pessoas.

33. Sei criar categorias de informação por interesse no computador.

34. Uso as tecnologias de que disponho para auxiliar na tomada de decisões.

35. Sei imprimir textos usando o computador. 
36. Evito baixar cópias de músicas sem fazer o pagamento, quando necessário.

37. Sei organizar dados por categorias, usando o computador.

38. Acesso sites.

39. Uso recursos de informática para me auxiliar na tomada de decisões.

40. Evito fazer cópias, para outras pessoas, dos cds que comprei.

4I. Consulto catálogo telefônico pela Internet.

42. Uso telefone celular.

43. Uso o computador para criar gráficos.

44. Pesquiso na Internet antes de comprar nas lojas.

45. Considero questões éticas quando faço uso da tecnologia.

46. Procuro saber o número de telefone dos meus colegas e informar o meu para eles.

47. Faço um itinerário usando os mapas disponíveis na Internet.

48. Se necessário, sei escolher equipamentos que melhoram o funcionamento das tarefas que realizo.

49. Procuro divulgar minhas descobertas com outras pessoas através da Internet.

50. Uso a Internet para tornar os gastos com telecomunicações mais baratos.

51. Sei dimensionar o computador que necessito para uso pessoal.

52. Sei identificar novas formas de utilizar os recursos tecnológicos de que disponho.

53. Uso a Internet para fazer minhas transações bancárias.

54. Participo de atividades da escola colaborando com meus colegas pela Internet.

55. Uso a Internet para fazer compras de forma on-line.

56. Produzo vídeos usando o computador.

57. Uso recursos extras do celular (câmera, gravador e outros).

58. Uso a Internet para pesquisar informações de diferentes fontes.

59. Uso o computador para criar tabelas.

60. Sei detalhes técnicos necessários para me conectar à Internet

61. Procuro atualizar meus conhecimentos usando a Internet.

62. Sei editar textos usando o computador.

63. Evito divulgar informações preconceituosas quando as vejo nos meios de comunicação.

64. Participo de uma comunidade virtual.

65. Evito fazer cópias, para outras pessoas, dos programas de computador que comprei.

66. Meus colegas e eu trabalhamos juntos utilizando a Internet para nos comunicarmos.

67. Sei resolver problemas técnicos básicos que possam ocorrer quando uso o computador.

68. Utilizo a Internet para me ajudar em minhas atividades de estudo.

69. Envio e-mails.

70. Sou capaz de debater idéias com outras pessoas através da Internet.

71. Desconfio de mensagens que recebo por e-mail e que não conheço a origem.

72. Eu crio apresentações com imagens e sons para trabalhos escolares.

73. Sei utilizar mecanismos de busca na internet para filtrar somente as informações que desejo.

74. Entendo o que o pessoal especializado em tecnologia diz.

75. Jogo usando o computador.

76. Sou capaz de adaptar as informações que preciso apresentar às pessoas, para que elas compreendam melhor o que tenho a dizer.

77. Uso o MSN.

78. Procuro seguir as recomendações da netiqueta (etiqueta na Internet).

79. Repasso informações aos meus colegas quando descubro algo novo.

80. Inscrevo-me em comunidades virtuais que são relacionadas à minha área de interesse.

81. Avalio as notícias que recebo pelos meios de comunicação antes de aceitá-las como verdades.

82. Tomo decisões sobre metas pessoais influenciado pelos meios de comunicação. 
83. Sei organizar listas de endereços dos sites que me interessam.

84. Aprendo mais rápido o que estou estudando quando uso o computador.

85. Uso o computador para analisar informações de pesquisa.

86. Sei instalar programas que garantam a segurança do meu computador.

87. Uso programas que protegem meu computador contra invasões ou divulgação de minhas informações sigilosas.

88. Participo de comunidades de estudo na Internet.

89. Faço pesquisa avançada para encontrar o que procuro, usando bases de dados.

90. Deleto mensagens desconhecidas que podem representar risco.

91. Conheço a legislação referente à informática.

92. Ajudo a atualizar conteúdo de sites, dando opinião ou enviando informação.

93. Sei escolher equipamentos de áudio e vídeo de que necessito.

94. Procuro atualizar meus conhecimentos usando a TV.

95. Uso a Internet para pesquisar temas internacionais.

Recebido em: |4/I2/2005

Revisado em: 24/04/2006

Aprovado em: 18/05/2006

\section{Sobre os autores:}

Maria Cristina Rodrigues Azevedo Joly (cristina.joly@saofrancisco.edu.br). Docente do curso de Psicologia e Pedagogia e do Programa de Pósgraduação stricto-sensu da Universidade São Francisco.

Ronei Ximenes Martins (ronei.ximenes@saofrancisco.edu.br ). Doutorando do Programa de Pós-graduação stricto sensu em Psicologia da Universidade São Francisco. Coordenador do Núcleo de Educação à Distância da Universidade São Francisco.

Endereço para correspondência

Rua Alexandre Rodrigues Barbosa, 45

|325|-900- Itatiba/SP 